\title{
MODELING MASONRY WITH LIMIT ANALYSIS FINITE ELEMENTS: REVIEW, APPLICATIONS AND NEW DIRECTIONS
}

\author{
P. B. Lourenço ${ }^{1}$ G. Milani ${ }^{2}$ \\ ${ }^{1}$ Department of Civil Engineering, School of Engineering \\ University of Minho \\ Azurém,4800-058 Guimaraes, Portugal \\ e-mail: pbl@civil.uminho.pt \\ ${ }^{2}$ Deparment of Engineering \\ Universy of Ferrara \\ Via Saragat 144100 Ferrara, Italy \\ e-mail: gmilani@ing.unife.it
}

Key words: Masonry, Limit analysis, Constitutive behavior, Homogenization techniques.

\begin{abstract}
The mechanics of masonry structures have been for long underdeveloped in comparison with other fields of knowledge. Presently, non-linear analysis is a popular field in masonry research and homogenization techniques play a major role, despite the mathematical and conceptual difficulties inherent to the approach.

Here, a simple micro-mechanical model for the homogenized limit analysis of masonry is reviewed. Assuming brickwork under plane stress condition and adopting a polynomial expansion for the stress field, a linear optimization problem is derived on the elementary cell in order to recover the homogenized failure surface of the brickwork. The implementation of the homogenized failure surfaces for inplane and out-of-plane behaviour in a finite element limit analysis code is addressed. Relevant structural examples are treated with particular emphasis on the upper bound method, and are compared with competing approaches. Finally, the aspects of reliability and safety of masonry structures are discussed using the proposed modelling strategy.
\end{abstract}

Keywords: Masonry, Limit analysis, Constitutive behaviour, Homogenization techniques.

\section{Introduction}

Masonry is a composite material constituted by units jointed together with mortar. Units are such as bricks, blocks, ashlars, adobes, irregular stones and others. Mortar can be clay, bitumen, chalk, lime/cement based mortar, glue or other. The huge number of possible combinations generated by the geometry, nature and arrangement of units as well as the characteristics of mortars raises doubts about the accuracy of the term "masonry". Still, much information can be gained from the 
study of regular masonry structures, in which a periodic repetition of the microstructure occurs due to a constant arrangement of the units (or constant bond).

The difficulties in performing advanced testing of this type of structures are quite large due to the innumerable variations of masonry, the large scatter of in situ material properties and the impossibility of reproducing it all in a specimen. Therefore, most of the advanced experimental research carried out in the last decades concentrated in brick / block masonry and its relevance for design. Accurate modelling requires a comprehensive experimental description of the material, which seems mostly available at the present state of knowledge, see Lourenço [1] [2] for a review.

Another important aspect that deserves particular consideration in the study of brickwork structures is related to the fact that historical city centers are often constituted by ancient masonry buildings. Many efforts have been done by researchers in order to better understand their behavior to horizontal seismic actions. For instance, earthquake surveys have demonstrated that the low tensile strength of masonry elements combined with an insufficient connection between perpendicular walls leads to overturning premature collapses of the perimeter walls under seismic horizontal acceleration and combined in- and out-of-plane failures [3].

Therefore, the evaluation of the ultimate load bearing capacity of masonry buildings subjected to horizontal loads is a fundamental task in their design and safety assessment. Simplified limit analysis methods are usually adopted by practitioners for safety analyses and design of strengthening [4], but codes of practice, as for instance the recent Italian O.P.C.M. 3431 [5] [6], require a static non linear analysis for existing masonry buildings, in which a limited ductile behavior of the elements is taken into account, featuring failure mechanisms such as rocking, shear and diagonal cracking of the walls.

In this framework, many researchers tried to propose a number of different numerical approaches (see [2] for a comprehensive review), all based on micromodeling, macro-modeling or homogenisation, with the aim of obtaining reliable tools to predict masonry behaviour at failure.

The present paper focuses exclusively on the analysis of masonry structures making use of homogenization techniques, which has been receiving a growing interest from the scientific community.

In particular, the approach based on the use of averaged constitutive equations seems to be the only one suitable to be employed in a large scale finite element analysis [7]. In fact, heterogeneous approaches based on a distinct representation of bricks and joints seem to be limited to the study of panels of small dimensions, due to the large number of variables involved in a non linear finite element analysis. On the other hand, alternative strategies based on macro-modeling (see Lourenço et al. [8]) have the drawback of requiring a preliminary mechanical characterization of the model, which usually is obtained from experimental data fitting [9].

In this framework, homogenization techniques can be used for the analysis of large scale structures. Such techniques take into account at a cell level the mechanical properties of constituent materials and the geometry of the elementary cell, allowing the analysis of entire buildings through standard finite element codes. Furthermore, the application of homogenization theory to the rigid-plastic case [10] 
is particularly indicated for a simple but reliable structural analysis, requiring only a reduced number of material parameters and providing significant information at failure, such as limit multipliers, collapse mechanisms and, at least on critical sections, the stress distribution [11].

In this paper, the micro-mechanical model presented by the authors in [11] [12] and [13] for the limit analysis of respectively in- and out-of-plane loaded masonry walls is extended and utilized in presence of coupled membrane and flexural effects (see also [14]). In the model, the elementary cell is subdivided along its thickness in several layers. For each layer, fully equilibrated stress fields are assumed, adopting polynomial expressions for the stress tensor components in a finite number of subdomains. The continuity of the stress vector on the interfaces between adjacent subdomains and suitable anti-periodicity conditions on the boundary surface are further imposed. In this way, linearized homogenized surfaces in six dimensions (polytopes) for masonry in- and out-of-plane loaded are obtained. Such surfaces are then implemented in a FE limit analysis code for the analysis at collapse of entire 3D structures and meaningful examples of technical relevance are discussed in detail.

In Section 2, a micro-mechanical model for obtaining masonry homogenized inand out-of-plane failure surfaces is presented, whereas in Section 3 a 3D FE upper bound approach suitable for the analysis at collapse of entire buildings is presented. The method is based on a triangular discretization of the structure, so that the velocity field interpolation is linear inside each element. Plastic dissipation can occur for in-plane actions both in continuum and in interfaces. Being the velocity interpolation linear inside each element, the curvature rate tensor is equal to zero for each triangle and out-of-plane dissipation can take place only at the interfaces between adjoining triangles.

In Section 4, a large scale masonry building subjected to seismic loads is numerically analyzed with the model proposed. Then, a sensitivity analysis is conducted, varying in a wide range mortar cohesion and friction angle.

Finally, in Section 5, some basic aspects connected to reliability and safety concepts are discussed using the proposed modeling strategy for a masonry shear wall in which the mechanical properties of joints are assumed as stochastic variables.

\section{In- and out-of-plane homogenized failure surfaces}

Let us consider a masonry wall $\Omega$ constituted by a periodic arrangement of bricks and mortar disposed in running bond, as shown in Figure 1-a.

As pointed out by Suquet in [10] for a general rigid-plastic heterogeneous material, homogenization techniques combined with limit analysis can be applied for the evaluation of the homogenized in- and out-of-plane strength domain $S^{\text {hom }}$, being masonry only a particular case of interest.

In the framework of perfect plasticity and associated flow rule for the constituent materials, and by means of the lower bound limit analysis theorem, $S^{\text {hom }}$ can be derived by means of the following (non-linear) optimization problem (see also Figure 1): 


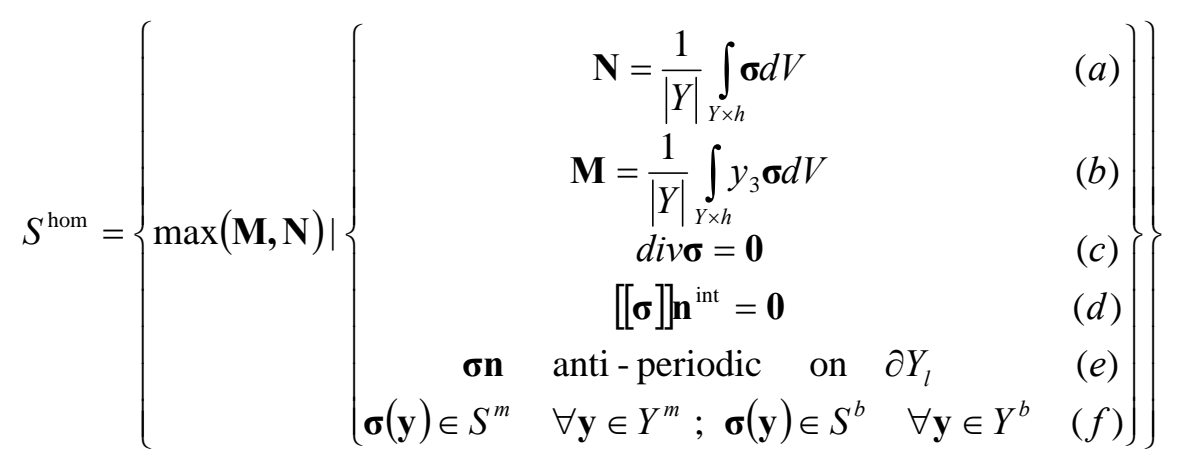

where:

- $\mathbf{N}$ and $\mathbf{M}$ are the macroscopic in-plane (membrane forces) and out-of-plane (bending moments and torsion) tensors;

- $\boldsymbol{\sigma}$ denotes the microscopic stress tensor;

- $\mathbf{n}$ is the outward versor of $\partial Y_{l}$ surface, see Figure 1-a;

- $[[\boldsymbol{\sigma}]]$ is the jump of micro-stresses across any discontinuity surface of normal $\mathbf{n}^{\text {int }}$, Figure 1-c;

- $S^{m}$ and $S^{b}$ denote respectively the strength domains of mortar and bricks;

- $Y$ is the cross section of the 3D elementary cell with $y_{3}=0$ (see Figure 1 ), $|Y|$ is its area, $V$ is the elementary cell volume, $h$ represents the wall thickness and $\mathbf{y}=\left(\begin{array}{lll}y_{1} & y_{2} & y_{3}\end{array}\right)$ are the assumed material axes;

- $Y^{m}$ and $Y^{b}$ represent mortar joints and bricks respectively, see Figure 1.

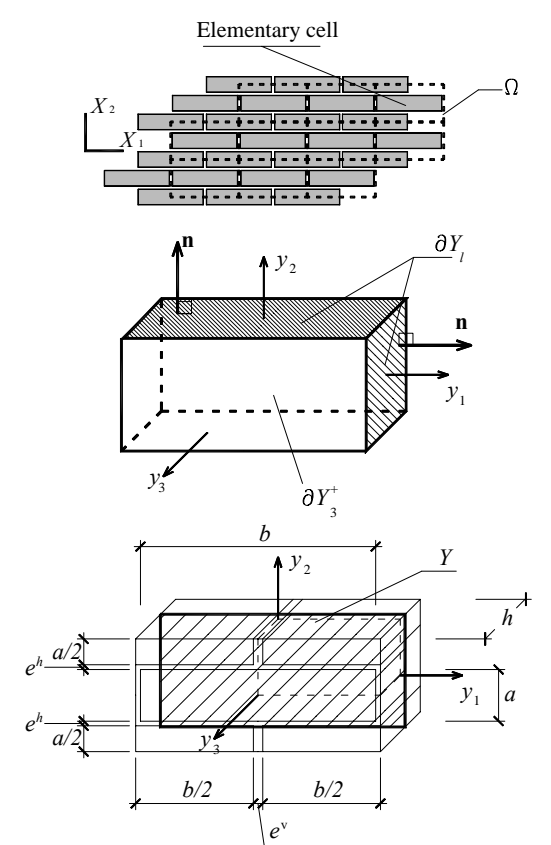

$-\mathrm{a}$

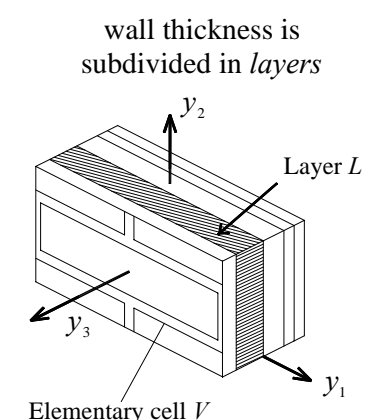

Elementary cell $V$

Each layer is subdivided in 36 sub-domains

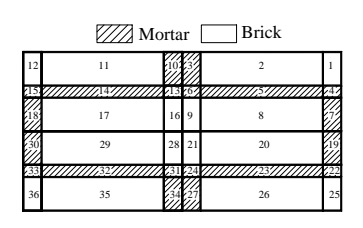

-b
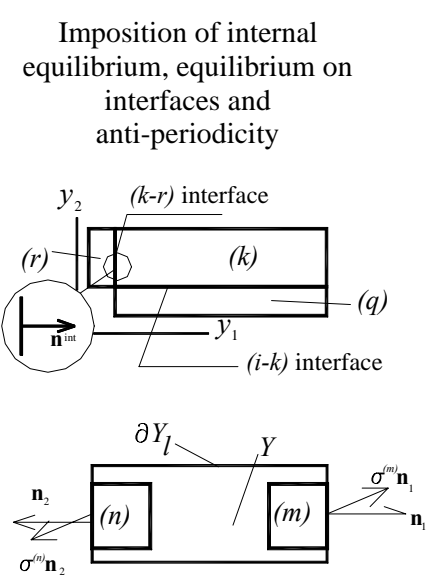

Figure 1: Proposed micro-mechanical model. -a: elementary cell. -b: subdivision in layers along thickness and subdivision of each layer in sub-domains. -c: imposition of internal equilibrium, equilibrium on interfaces and anti-periodicity. 
It is worth noting that condition ( 1-c ) imposes the micro-equilibrium with zero body forces, usually neglected in the framework of the homogenization theory and that anti-periodicity condition (1-e ) requires that that stress vectors $\boldsymbol{\sigma n}$ are opposite on opposite sides of $\partial Y_{l}$, Figure 1-c, i.e. $\sigma^{(m)} \mathbf{n}_{1}=-\sigma^{(n)} \mathbf{n}_{2}$

In order to solve Eqs. ( 1 ) numerically, the simple admissible and equilibrated micro-mechanical model proposed in [12] and [14] is adopted. The unit cell is subdivided into a fixed number of layers along its thickness, as shown in Figure 1-b. For each layer out-of-plane components $\sigma_{i 3}(i=1,2,3)$ of the micro-stress tensor $\boldsymbol{\sigma}$ are set to zero, so that only in-plane components $\sigma_{i j}(i, j=1,2)$ are considered active. Furthermore, $\sigma_{i j}(i, j=1,2)$ are kept constant along the $\Delta_{L}$ thickness of each layer, i.e. in each layer $\sigma_{i j}=\sigma_{i j}\left(y_{1}, y_{2}\right)$. For each layer in the wall thickness direction, one-fourth of the representative volume element is sub-divided into nine geometrical elementary entities (sub-domains), so that the entire elementary cell is sub-divided into 36 sub-domains (see [12] for further details and Figure 1-b).

For each sub-domain $(k)$ and layer $(L)$, polynomial distributions of degree $(m)$ in the variables $\left(y_{1}, y_{2}\right)$ are a priori assumed for the stress components. Since stresses are polynomial expressions, the generic $i j$ th component can be written as follows:

$$
\sigma_{i j}^{(k, L)}=\mathbf{X}(\mathbf{y}) \mathbf{S}_{i j}^{(k, L) T} \quad \mathbf{y} \in Y^{(k, L)}
$$

where:

- $\mathbf{X}(\mathbf{y})=\left[\begin{array}{lllllll}1 & y_{1} & y_{2} & y_{1}^{2} & y_{1} y_{2} & y_{2}^{2} & \ldots\end{array}\right]$;

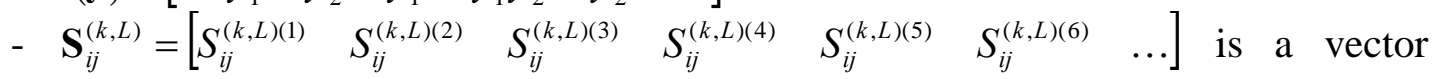
representing the unknown stress parameters of sub-domain $(k)$ of layer $(L)$;

- $Y^{(k, L)}$ represents the $k$ th sub-domain of layer $(L)$.

The imposition of equilibrium inside each sub-domain, the continuity of the stress vector on interfaces and the anti-periodicity of $\boldsymbol{\sigma n}$ permit a reduction in the number of independent stress parameters. For the sake of conciseness, the reader is referred to [12] for further details.

Assemblage operations on the local variables allow to write the stress vector $\tilde{\boldsymbol{\sigma}}^{(k, L)}$ of layer $L$ inside each sub-domain as:

$$
\tilde{\boldsymbol{\sigma}}^{(k, L)}=\tilde{\mathbf{X}}^{(k, L)}(\mathbf{y}) \tilde{\mathbf{S}}^{(L)} \quad k=1, \ldots, \text { no. of sub-domains } L=1, \ldots \text {, no. of layers }
$$

where $\tilde{\mathbf{S}}^{(L)}$ is a $N_{u k} x 1$ ( $N_{u k}=$ number of unknowns per layer) vector of linearly independent unknown stress parameters of layer $L$ and $\tilde{\mathbf{X}}^{(k, L)}(\mathbf{y})$ is a $3 x N_{u k}$ matrix depending only on the geometry of the elementary cell and on the position $\mathbf{y}$ of the point in which the micro-stress is evaluated.

For out-of-plane actions the proposed model requires a subdivision $\left(n_{L}\right)$ of the wall thickness into several layers (Figure 1-b), with a fixed constant thickness 
$\Delta_{L}=h / n_{L}$ for each layer. This allows to derive the following simple (non) linear optimization problem:

$$
S^{\mathrm{hom}} \equiv\left\{\text { such that } \left\{\begin{array}{cc}
\max \{\lambda\} & (a) \\
\tilde{\mathbf{N}}=\int_{k, L} \tilde{\boldsymbol{\sigma}}^{(k, L)} d V & (b) \\
\tilde{\mathbf{M}}=\int_{k, L}^{k} y_{3} \tilde{\boldsymbol{\sigma}}^{(k, L)} d V & (c) \\
\boldsymbol{\Sigma}=[\tilde{\mathbf{N}} \tilde{\mathbf{M}}]=\lambda \mathbf{n}_{\Sigma} & (d) \\
\tilde{\boldsymbol{\sigma}}^{(k, L)}=\tilde{\mathbf{X}}^{(k, L)}(\mathbf{y}) \tilde{\mathbf{S}} & (e) \\
\tilde{\boldsymbol{\sigma}}^{(k, L)} \in S^{(k, L)} & (f) \\
k=1, \ldots, \text { number of sub-domains } & (g) \\
L=1, \ldots, \text { number of layers } &
\end{array}\right.\right.
$$

where:

- $\lambda$ is the load multiplier (ultimate moment, ultimate membrane action or a combination of moments and membrane actions) with fixed direction $\mathbf{n}_{\Sigma}$ in the six dimensional space of membrane actions $\left(\tilde{\mathbf{N}}=\left[N_{x x} N_{x y} N_{y y}\right]\right)$, together with bending and torsion moments $\left(\tilde{\mathbf{M}}=\left[M_{x x} M_{x y} M_{y y}\right]\right.$ ).

- $S^{(k, L)}$ denotes the (non-linear) strength domain of the constituent material (mortar or brick) corresponding to the $k$ th sub-domain and $L$-th layer.

- $\tilde{\mathbf{S}}$ collects all the unknown polynomial coefficients (of each sub-domain of each layer).

It is noted that the direction $\mathbf{n}_{\Sigma}$ is fixed arbitrarily in the six dimensional space $\left[\begin{array}{ll}\tilde{\mathbf{N}} & \tilde{\mathbf{M}}\end{array}\right]$. As a rule, since $\mathbf{n}_{\Sigma}=\left[\alpha_{1}, \alpha_{2}, \ldots, \alpha_{6}\right]$ with $\Sigma \alpha_{i}^{2}=1$, the parameters $\alpha_{i}$ are chosen randomly between 0 to 1 satisfying the constraint $\Sigma \alpha_{i}^{2}=1$, so that a number of directions $\mathbf{n}_{\Sigma}$ are selected.

In what follows, wall thickness is subdivided into thirty layers. The authors experienced that more refined discretizations result in negligible changes in the homogenized failure surface.

\section{3D kinematic FE limit analysis: basic assumptions}

The upper bound approach developed in this paper is based both on the formulation presented in Sloan and Kleeman [15] for the in-plane case and on the formulation by Munro and Da Fonseca [16] for out-of-plane actions, also adopted in [17].

The formulation uses three noded triangular elements with linear interpolation of the velocity field inside each element, so that three velocity unknowns per node $i$, say $w_{x x}^{i}, w_{y y}^{i}$ and $w_{z z}^{i}$ (respectively 2 in-plane velocities and 1 out-of-plane velocity, see Figure 2-a) are introduced for each element $E$, meaning that the velocity field is linear inside an element, whereas the strain rate field is constant for in-plane actions. 
For the sake of simplicity, it is assumed that jump of velocities on interfaces occurs only in the plane containing two contiguous and coplanar elements, with linear interpolation of the jump along the interface. Hence, for each interface between coplanar adjacent elements, four additional unknowns are introduced ( $\Delta \mathbf{u}^{I}=\left[\Delta v_{1} \Delta u_{1} \Delta v_{2} \Delta u_{2}\right]^{T}$ ), representing the normal $\left(\Delta v_{i}\right)$ and tangential $\left(\Delta u_{i}\right)$ jumps of velocities (with respect to the discontinuity direction) evaluated on nodes $i=1$ and $i=2$ of the interface (see Figure 2-b). For the sake of simplicity, it is assumed in the model that, if two adjacent elements do not lay in the same plane, no discontinuity occurs between the velocities belonging to the elements, so a priori assuming a perfect interlocking between perpendicular walls.

Hence, for any pair of nodes on the interface between two adjacent and coplanar triangles $R$ and $K$, the tangential and normal velocity jumps can be written in terms of the Cartesian nodal velocities of elements $R-K$ (see [15] for details), so that four linear equations in the following form can be written:

$$
\mathbf{A}_{11}^{e q} \mathbf{w}^{R}+\mathbf{A}_{12}^{e q} \mathbf{w}^{K}+\mathbf{A}_{13}^{e q} \Delta \mathbf{u}^{I}=\mathbf{0}
$$

where $\mathbf{w}^{R}$ and $\mathbf{w}^{K}$ are the $9 \times 1$ vectors that collect velocities of elements $R$ and $K$ respectively and $\mathbf{A}_{1 j}^{e q} \quad j=1,2,3$ are matrices which depend only on the interface orientation $\Omega^{I}$ (Figure 2).

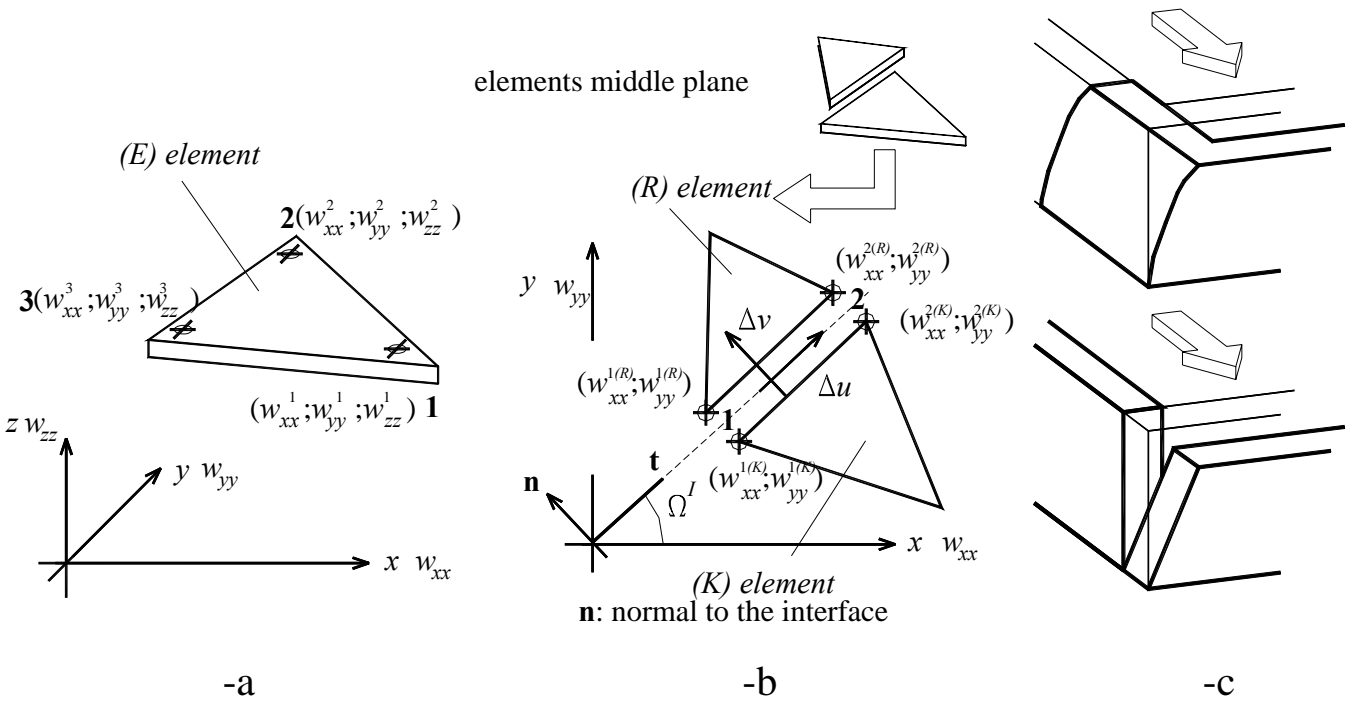

Figure 2: -a: Triangular plate and shell element used for the upper bound FE limit analysis. -b: discontinuity of the in-plane velocity field. -c: perfect interlocking and absence of connections between perpendicular walls.

Under in-plane loads three equality constrains representing the plastic flow in continuum (obeying an associated flow rule) are introduced for each element in the form $\dot{\boldsymbol{\varepsilon}}_{p l}^{E}=\dot{\lambda}^{E} \partial S^{\text {hom }} / \partial \boldsymbol{\Sigma}$, where $\dot{\boldsymbol{\varepsilon}}_{p l}^{E}$ is the plastic strain rate vector of element $E$, $\dot{\lambda}^{E} \geq 0$ is the plastic multiplier, $S^{\text {hom }}$ is the homogenized (non) linear failure polytope of masonry and $\Sigma$ is the vector of macroscopic variables $\boldsymbol{\Sigma}=\left(N_{11}, N_{12}, N_{22}, M_{11}, M_{12}, M_{22}\right)$. 
We refer the reader to the previous section and to [11] for further details on the procedure used for obtaining a linear approximation (with $m$ hyper-planes) of the failure polytope in the form $S^{\text {hom }} \equiv \mathbf{A}^{i n} \boldsymbol{\Sigma} \leq \mathbf{b}^{\text {in }}$, where $\mathbf{A}^{\text {in }}$ is a $m \times 6$ matrix of coefficients of each hyper-plane and $\mathbf{b}^{\text {in }}$ is a $m \times 1$ vector of the right hand sides of the linear approximation.

Here we only recall that three linear equality constraints per element can be written $\left(\mathbf{A}_{11}^{e q} \mathbf{w}^{E}+\mathbf{A}_{12}^{e q} \dot{\lambda}^{E}=\mathbf{0}\right.$, where $\mathbf{w}^{E}$ is the vector of element velocities and $\dot{\lambda}^{E}$ is a $m \times 1$ vector of plastic multiplier rates, one for each plane of the linearised failure surface).

As stated by Munro and Da Fonseca [16], out-of-plane plastic dissipation occurs only along each interface $I$ between two adjacent triangles $R$ and $K$ or on a boundary side $B$ of an element $Q$ (see Figure 3 ).

Denoting with $\mathbf{w}_{z z, E}=\left[\begin{array}{llll}w_{z z}^{i(E)} & w_{z z}^{j(E)} & w_{z z}^{k(E)}\end{array}\right]^{T}$ the element $E$ out-of-plane nodal velocities and with $\dot{\boldsymbol{\theta}}_{E}=\left[\begin{array}{lll}\dot{\vartheta}_{i}^{E} & \dot{\vartheta}_{j}^{E} & \dot{\vartheta}_{k}^{E}\end{array}\right]^{T}$ the side normal rotation rates, it is possible to show that $\dot{\boldsymbol{\theta}}_{E}$ and $\mathbf{w}_{z z, E}$ are linked by the compatibility equation (Figure 3) $\dot{\boldsymbol{\theta}}_{E}=\mathbf{B}_{E} \mathbf{w}_{z z, E}$, where $\mathbf{B}_{E}$ is a $3 \times 3$ matrix that depends only on the geometry of element $E$.

The total internal power dissipated $P^{\text {in }}$ is constituted by the power dissipated in continuum, $P_{E}^{i n}$, and the power dissipated on interfaces, $P_{I}^{i n}$.

$P_{E}^{i n}$ can be evaluated for each triangle $E$ of area $A_{E}$ taking into account that curvature rates $\dot{\chi}_{x x}, \dot{\chi}_{x y}, \dot{\chi}_{y y}$ are zero in continuum, so that the flexural part of the model does not dissipate power in the continuum. As the homogenized (linearised) failure surface is constituted by $m$ hyper-planes of equation $A_{x x}^{q} N_{x x}+A_{y y}^{q} N_{y y}+A_{x y}^{q} N_{x y}+B_{x x}^{q} M_{x x}+B_{y y}^{q} M_{y y}+B_{x y}^{q} M_{x y}=C_{E}^{q}$, with the subscript $q$ such that $1 \leq q \leq m$, an estimation of $P_{E}^{i n}$ can be obtained as $P_{E}^{i n}=A_{E} \sum_{q=1}^{m} C_{E}^{q} \dot{\lambda}_{E}^{(q)}$ with:

$$
\begin{aligned}
& \dot{\chi}_{r q}=\sum_{q=1}^{m} \dot{\lambda}_{E}^{(q)} \partial S^{\text {hom }} / \partial \Sigma_{M r q}=0 \\
& r q=x x, x y, y y \\
& \Sigma_{M r q}=M_{r q}
\end{aligned}
$$

where $\dot{\lambda}_{E}^{(q)}$ is the plastic multiplier rate of the triangle $E$ associated to the $q$ th hyper-plane of the linearised failure surface.

For an interface $I$ of length $\Gamma$ and orientation $\Omega^{I}$, a rotation operator is applied to the linearized homogenized failure surface in order to obtain with a few row operations $m$ equations (one for each hyper-plane) in the form $A_{t t}^{q} N_{t t}+A_{n n}^{q} N_{n n}+A_{t n}^{q} N_{t n}+B_{t t}^{q} M_{t t}+B_{n n}^{q} M_{n n}+B_{t n}^{q} M_{t n}=C_{I}^{q} \quad$ representing the homogenized failure surface $\widetilde{S}^{\text {hom }}$ in the $\mathbf{n}-\mathbf{t}$ interface frame of reference, Figure 2. 
Therefore, the power dissipated $P_{I}^{\text {in }}$ along an interface $I$ of length $\Gamma$ and with orientation $\Omega^{I}$ can be estimated as follows:

$$
\begin{array}{ll}
P_{I}^{i n}=\int_{\Gamma} \sum_{q=1}^{m} C_{I}^{q} \dot{\lambda}_{I}^{(q)}(\xi) d \xi & \dot{\vartheta}^{I}=\dot{\vartheta}_{i}^{R}+\dot{\vartheta}_{j}^{K} \\
\dot{\vartheta}_{n t}^{I}=\sum_{q=1}^{m} \dot{\lambda}_{E}^{(q)} \partial \tilde{S}^{\text {hom }} / \partial \Sigma_{M n t}=0 & \dot{\vartheta}_{t t}^{I}=\sum_{q=1}^{m} \dot{\lambda}_{E}^{(q)} \partial \tilde{S}^{\text {hom }} / \partial \Sigma_{M} \\
\Delta_{t t}^{I}=\sum_{q=1}^{m} \dot{\lambda}_{E}^{(q)} \partial \tilde{S}^{\text {hom }} / \partial \Sigma_{N t t}=0 & \dot{\vartheta}_{n n}^{I}=\sum_{q=1}^{m} \dot{\lambda}_{E}^{(q)} \partial \tilde{S}^{\text {hom }} / \partial \Sigma_{i}
\end{array}
$$

where:

- $\dot{\lambda}_{I}^{(q)}(\xi)$ represents the $q$ th plastic multiplier rate of a point $\xi$ of the interface $I$;

- $\dot{\vartheta}_{n t}^{I}$ is the torsional rotation rate between $R$ and $K$ along $I$ assumed equal to zero in the model, i.e. assuming out-of-plane dissipation due only to flexion;

- $\dot{\vartheta}_{t t}^{I}$ is the bending rotation rate between $R$ and $K$ on $I$ along $\mathbf{t}$, assumed equal to zero;

- $\Delta_{t t}^{I}$ represents the interface plastic strain rate of $I$ along $\mathbf{t}$, integrated along the infinitesimal thickness of the interface;

- $\Sigma_{M n t}, \Sigma_{M t t}$ and $\Sigma_{N t t}$ are the torsional moment on $I\left(\Sigma_{M n t}=M_{n t}\right)$, the bending moment perpendicular to $\mathbf{t}$ direction $\left(\Sigma_{M t t}=M_{t t}\right)$ and the in-plane action parallel to t $\left(\Sigma_{N t t}=N_{t t}\right)$ respectively;

- $\dot{\vartheta}_{n n}^{I}$ is the bending rotation rate between $R$ and $K$ on $I$ along $\mathbf{n}$, i.e. $\dot{\vartheta}^{I}$ (Figure 3).

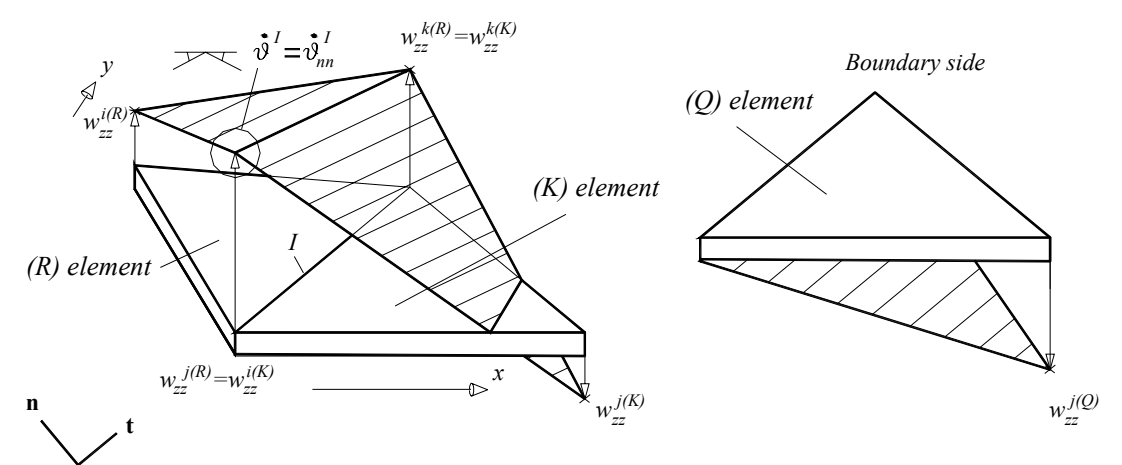

Figure 3: Rotation along an interface between adjacent triangles or in correspondence of a boundary side.

For what concerns external power dissipation, no differences occur with respect to classic FE limit analysis codes. External power dissipated can be written as $P^{e x}=\left(\mathbf{P}_{0}^{T}+\lambda \mathbf{P}_{1}^{T}\right) \mathbf{w}$, where $\mathbf{P}_{0}$ is the vector of (equivalent lumped) permanent loads, 
$\lambda$ is the load multiplier for the structure examined, $\mathbf{P}_{1}^{T}$ is the vector of (lumped) variable loads and $\mathbf{w}$ is the vector of assembled nodal velocities. As the amplitude of the failure mechanism is arbitrary, a further normalization condition $\mathbf{P}_{1}^{T} \mathbf{w}=1$ is usually introduced. Hence, the external power becomes linear in $\mathbf{w}$ and $\lambda$, i.e. $P^{e x}=\mathbf{P}_{0}^{T} \mathbf{w}+\lambda$.

After some assemblage operations (not reported here for the sake of conciseness), the following linear programming problem is obtained (analogous to that reported in [15], see also [18]-[20]), where the objective function consists in the minimization of the total internal power dissipated:

$$
\min \left\{\sum_{I=1}^{n^{I}} P_{I}^{i n}+\sum_{E=1}^{n^{E}} P_{E}^{i n}-\mathbf{P}_{0}^{T} \mathbf{w}\right\} \text { such that }\left\{\begin{array}{c}
\mathbf{A}^{e q} \mathbf{U}=\mathbf{b}^{e q} \\
\dot{\lambda}^{I, a s s} \geq \mathbf{0} \quad \dot{\lambda}^{E, a s s} \geq \mathbf{0} \\
\dot{\boldsymbol{\theta}}^{\text {ass }}=\dot{\boldsymbol{\theta}}^{+}-\dot{\boldsymbol{\theta}}^{-} \\
\dot{\boldsymbol{\theta}}^{+} \geq 0 \quad \boldsymbol{\theta}^{-} \geq 0
\end{array}\right.
$$

where:

- $\mathbf{U}$ is the vector of global unknowns and collects the vector of assembled nodal velocities ( $\mathbf{w}$ ), the vector of assembled element plastic multiplier rates $\left(\dot{\lambda}^{E, a s s}\right)$, the vector of assembled jump of velocities on interfaces $\left(\Delta \mathbf{u}^{I, \text { ass }}\right)$, the vector of assembled interface plastic multiplier rates $\left(\dot{\boldsymbol{\lambda}}^{I, \text { ass }}\right)$ and the vector of interface and boundary out-of-plane rotation angles $\dot{\boldsymbol{\theta}}^{\text {ass }}$;

- $\mathbf{A}^{e q}$ is the overall constraints matrix and collects normalization conditions, velocity boundary conditions, relations between velocity jumps on interfaces and elements velocities, constraints for plastic flow in velocity discontinuities and constraints for plastic flow in continuum.

- $n^{E}$ and $n^{I}$ are the total number of elements and interfaces, respectively.

\section{3D limit analysis of a school located in Italy}

The example treated in this Section consists of the prediction of the horizontal failure load of a three storey masonry building located in Ferrara, Italy, see Figure 4. The building, erected at the end of 19th century, is a school (A.Varano school) standing in Via Ghiara, Ferrara (Italy), in an isolated position and consists of two structurally independent rectangular main bodies, as shown in the plan view reported in Figure 5.

The main building, called here for the sake of simplicity Body A presents a rectangular shape with dimensions $\mathrm{L}_{1} \times \mathrm{L}_{2}=49.0 \times 12.2 \mathrm{~m}$ and 3 storeys, whereas the secondary Body $B$ has a rectangular shape $\mathrm{L}_{1} \times \mathrm{L}_{2}=8 \times 13 \mathrm{~m}$ and 3 storeys. All the walls are made with clay bricks, assumed of dimensions $250 \times 120 \times 55 \mathrm{~mm}$ (length $\times$ width $\times$ height) in absence of precise information. The first storey height is $4.85 \mathrm{~m}$ whereas the second and third storeys height is $4.65 \mathrm{~m}$. 


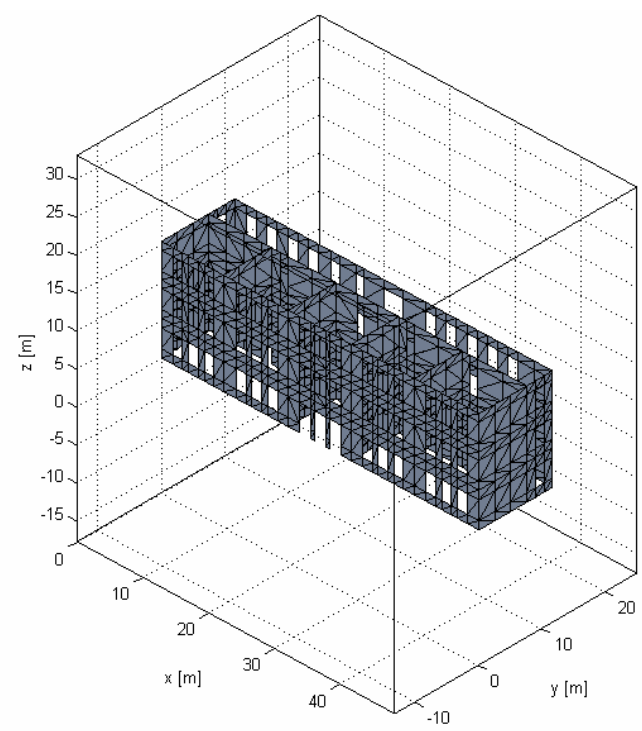

$-\mathrm{a}$

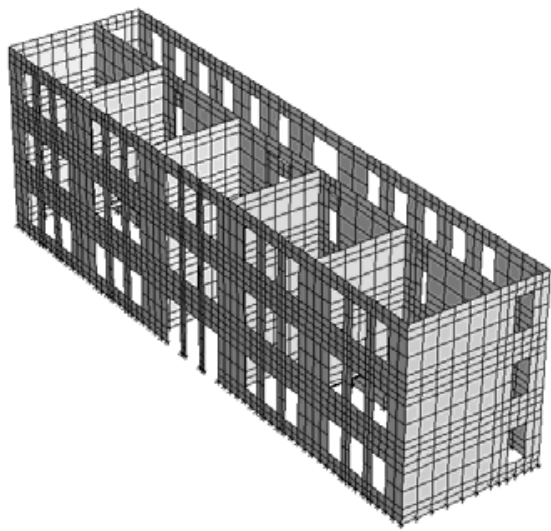

-b

Figure 4: Varano School. -a: Mesh used for the limit analysis (1576 triangular elements) and (-b) mesh used in Strand 7 for an elastic-plastic analysis with MohrCoulomb failure criterion (3152 plate elements).

In the intervention executed during the 1980's a $20 \mathrm{~mm}$ separation joint was introduced between Body A and B. Therefore, only Body A is taken here into consideration for the structural analysis.

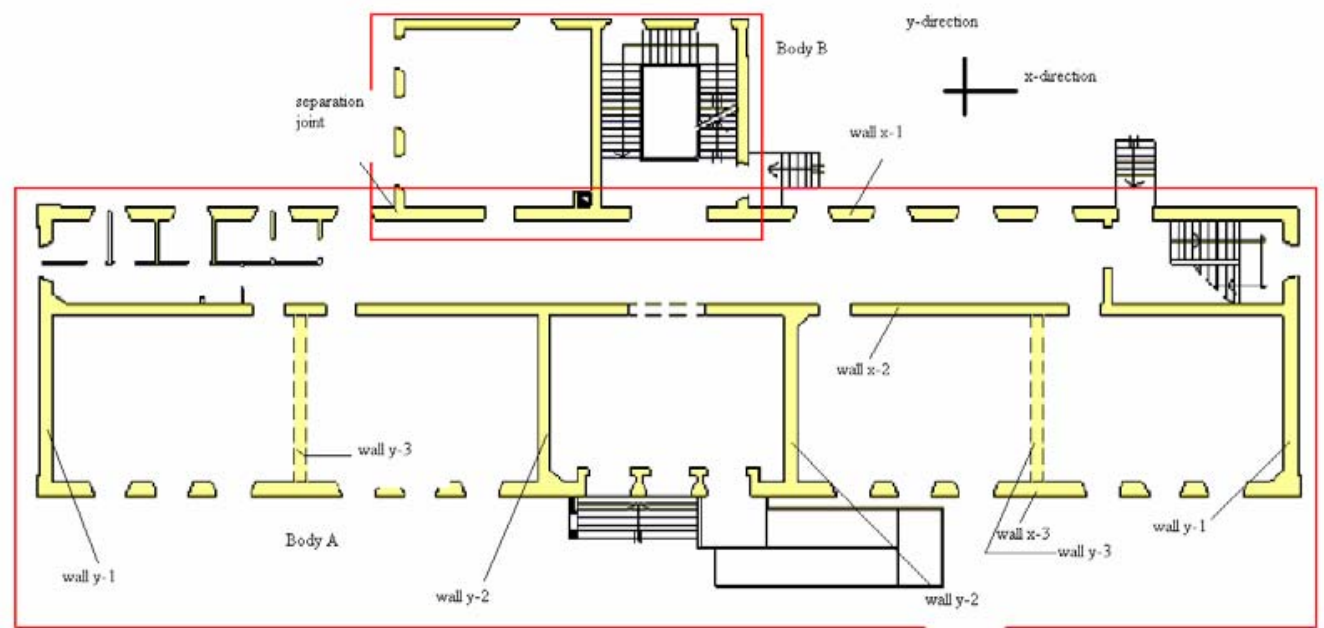

Figure 5: First floor plan view, Varano school.

Body A is geometrically regular with equally distributed mass, except for the large openings at the center of the first floor of the three walls parallel to $\mathrm{x}$ direction, which are part of a corridor giving access to the building. A main corridor of access to classrooms is located between walls $x-1$ and $x-2$, Figure 5 . Walls thickness is reported in Table I. 
Table I: Walls thickness (cm), Varano building.

\begin{tabular}{|c|c|c|c|c|c|c|}
\hline storey & $\mathrm{x}-1$ & $\mathrm{x}-2$ & $\mathrm{x}-3$ & $\mathrm{y}-1$ & $\mathrm{y}-2$ & $\mathrm{y}-3$ \\
\hline 1 & 60 & 45 & 60 & 60 & 45 & - \\
\hline 2 & 50 & 45 & 50 & 50 & 45 & 45 \\
\hline 3 & 45 & 30 & 45 & 45 & 30 & 30 \\
\hline
\end{tabular}

A FE model consisting of 1576 triangular elements is used for performing the homogenized limit analysis proposed (Figure 4-a) under a static equivalent seismic load directed along x-direction. The results obtained with the homogenized FE limit analysis model (i.e. failure shear at the base and failure mechanism) are compared with a standard FE elastic-perfectly plastic analysis conducted by means of a standard FE model (Strand 7). The analysis is performed using a mesh of 3152 four noded shell elements supposing masonry isotropic with a pure Mohr-Coulomb failure criterion.

For masonry, a cohesion $c$ equal to $0.12 \mathrm{~N} / \mathrm{mm}^{2}$ and friction angle $\Phi=\tan ^{-1}(0.4)$ are adopted for the simulations, in agreement with the Italian code [5] [6]. In order to compare the homogenized limit analysis procedure proposed with the standard FE model, a linearized Lourenço-Rots [21] [22] failure criterion for joints is adopted for the homogenization approach, whereas for units a cut-off failure criterion in compression is assumed, see Table II.
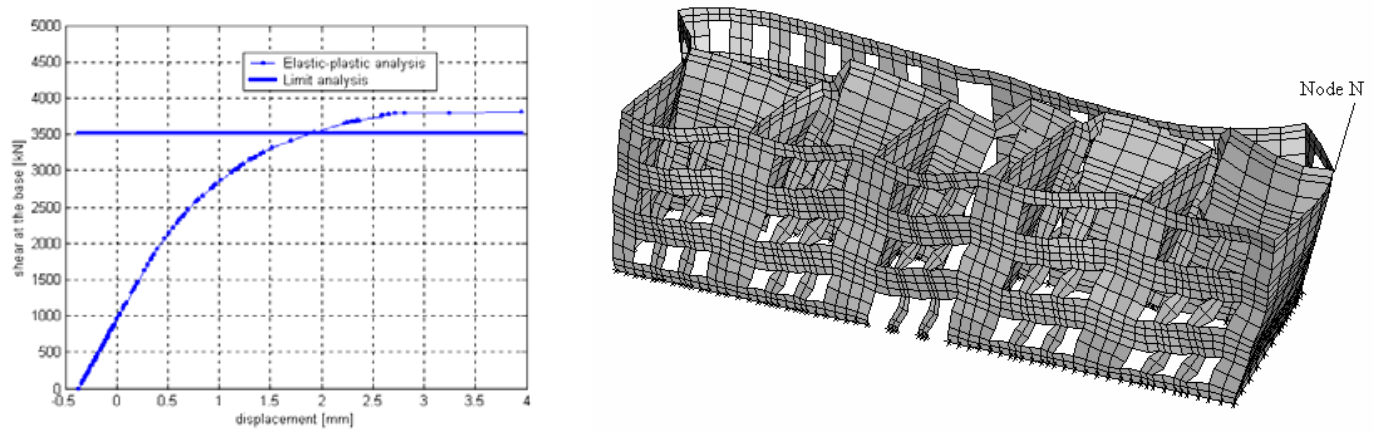

Figure 6: Varano school. Standard FE elastic plastic approach. -a: shear at the base node $\mathrm{N}$ displacement curve. -b: deformed shape at collapse.

Table II: Mechanical characteristics assumed for joints and bricks, Varano building. Here, $f_{t}$ is the tensile strength, $f_{c}$ is the compressive strength (compressive cut-off both for joints and bricks) $c$ is the cohesion and $\Phi_{1}$ is the friction angle.

\begin{tabular}{|c|c|c|c|c|c|c|}
\hline \multicolumn{3}{|c|}{ Joint } & \multicolumn{2}{c|}{ Brick } \\
\hline$c\left[N / \mathrm{mm}^{2}\right]$ & $f_{t}\left[N / \mathrm{mm}^{2}\right]$ & $f_{c}\left[N / \mathrm{mm}^{2}\right]$ & $\Phi_{1}$ & $f_{c}\left[\mathrm{~N} / \mathrm{mm}^{2}\right]$ \\
\hline 0.12 & 0.12 & 5 & $35^{\circ}$ & 30 \\
\hline
\end{tabular}


In both models, the seismic load is applied in correspondence of floor $i$ by means of a horizontal distributed load of intensity $k_{i} \hat{\lambda}$ ( $k_{i}$ non-dimensional constant), where $\hat{\lambda}$ is the collapse load and $k_{i}$ is taken, in agreement with the Italian code [5][23], equal to $z_{i} W_{i} /\left(\sum_{i}^{n} z_{i} W_{i}\right)$, where $W_{i}$ is the $i$ th floor vertical load, $z_{i}$ is the $i$ th floor distance to the ground and $n$ is the total number of floors.

Floors, constituted by small vaults made of clay bricks and supported by a framework of steel girders, are disposed parallel to y-direction in correspondence to the first and second floors and distribute vertical loads uniformly on $\mathrm{x}$-directed walls. At a first attempt, floors stiffness is not taken into account in the numerical model and vertical loads, which are independent from the load multiplier, are applied directly on masonry walls in correspondence to the floors. In correspondence of the third floor, a timber truss structure supports an inclined roof covering. For the sake of simplicity, self weight of masonry is supposed concentrated in correspondence to the floors and added to the remaining dead loads, which are defined according to the Italian code [23] (see also [24] and [25]).
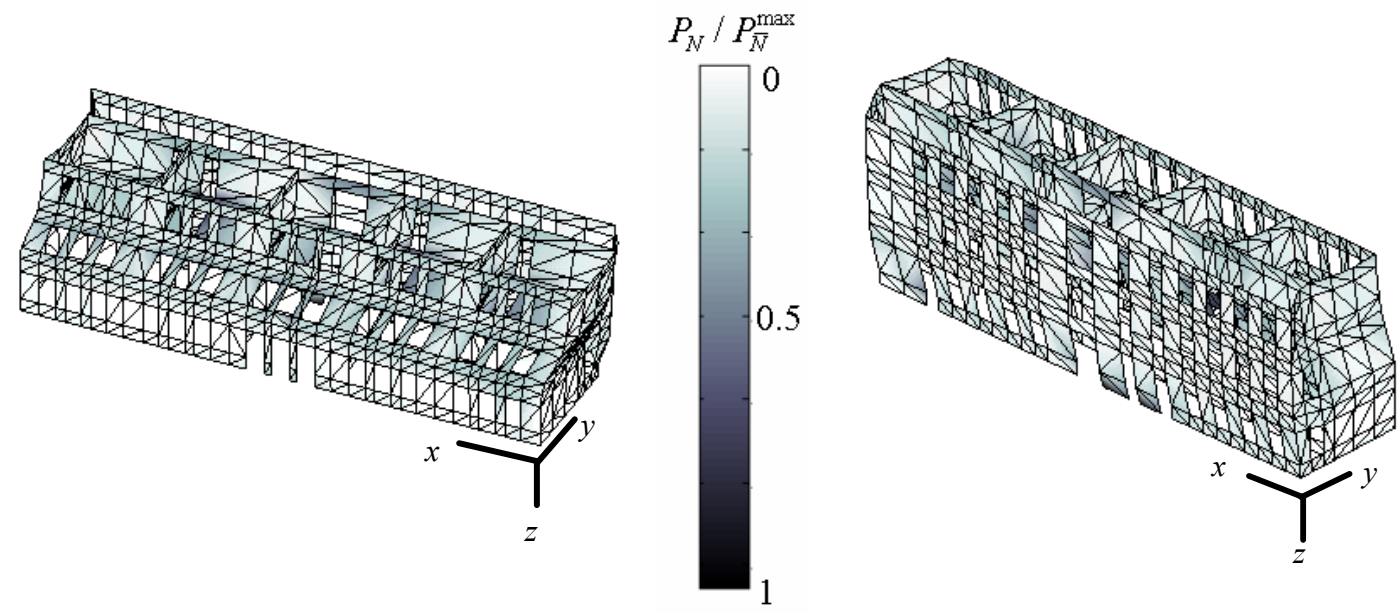

Figure 7: Varano school. Failure mechanism A and concentration of plastic dissipation for the entire building, homogenization FE limit analysis approach. $P_{N}$

is the in-plane plastic dissipation evaluated at node $N$ and $\bar{N}$ is the node of maximum dissipation.

The kinematic FE homogenized limit analysis gives a total shear at the base of the building of $3520 \mathrm{kN}$, in good agreement with the results obtained with the standard FE procedure. In this case, in fact, the capacity curve of the building, Figure 6-a, reaches its maximum at approximately $3800 k N$. Finally, the deformed shape at collapse of both models, compare Figure 6-b and Figure 7, demonstrates that a combined in- and out-of-plane failure takes place and that failure is mainly concentrated along walls $\mathrm{x}-2$ and $\mathrm{x}-3$.

A sensitivity analysis is finally conducted, assuming for joints a classic MohrCoulomb failure criterion with tension cut-off $f_{t}$ equal to 
$\min \left\{0.05 \mathrm{~N} / \mathrm{mm}^{2} \quad \mathrm{c} / \tan \Phi\right\}$, compressive cut-off $f_{t}=5 \mathrm{~N} / \mathrm{mm}^{2}$ and varying cohesion $c$ and friction angle $\Phi$ in the range $0.01-0.5 \mathrm{~N} / \mathrm{mm}^{2}$ and 5-35 . For bricks a limited compressive strength equal to $30 \mathrm{~N} / \mathrm{mm}^{2}$ is also assumed.

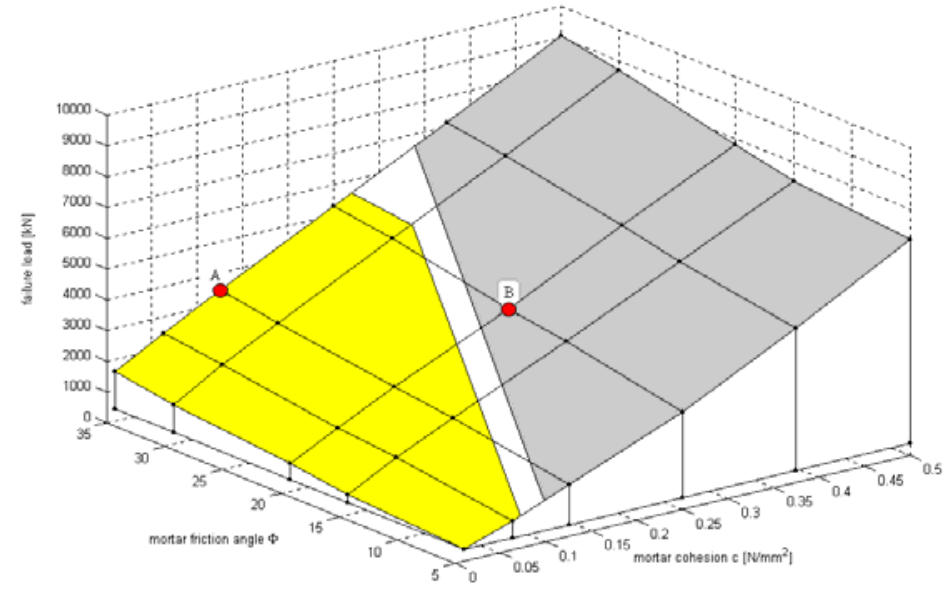

Figure 8: Varano school. Sensitivity analysis varying mortar cohesion and mortar friction angle and failure mechanisms patch.

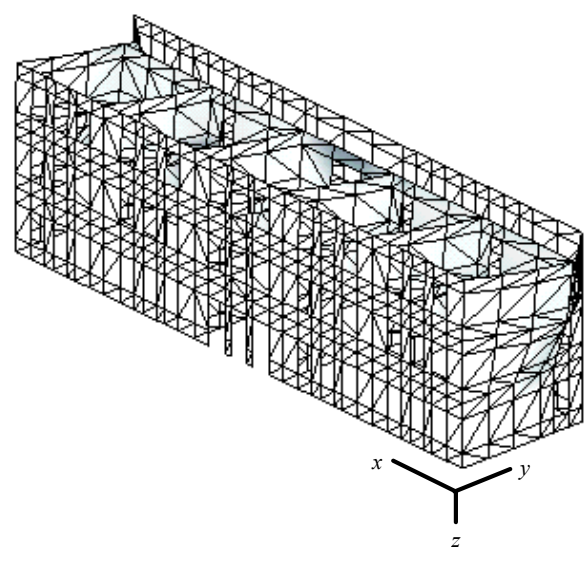

Failure mechanism B, view 1

$-\mathrm{a}$

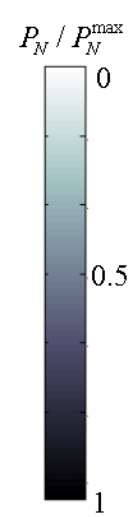

Failure mechanism B, view 2 .

-b

Figure 9: Varano school. Failure mechanisms B. $P_{N}$ is the in-plane plastic

dissipation evaluated at node $N$ and $\bar{N}$ is the node of maximum dissipation.

In Figure 8, the failure load of the structure is reported varying mortar cohesion and friction angle. Two different failure mechanisms can be distinguished, labelled as failure mechanism $\mathrm{A}$ and $\mathrm{B}$. The intervals in which they take place are indicated in Figure 8. In particular, mechanisms A, reported in Figure 7, corresponds to an inplane failure of walls $\mathrm{x}-2$ and $\mathrm{x}-3$ combined with an out-of-plane failure of walls $\mathrm{y}$ - 
1. On the other hand, Mechanism B, reported in Figure 9, combines a shear failure of wall $\mathrm{x}-2$ concentrated on the second storey and overturning of walls $\mathrm{y}-1$.

\section{$5 \quad$ Limit analysis of a shear masonry wall with stochastic mortar mechanical properties}

As a matter of fact, experimental laboratory tests reveal that the determination of mechanical properties of bricks and mortar are affected by large uncertainties. This implies that different masonry structures, even if constructed under the same nominal conditions, could show quite different ultimate strength.

In this section, a homogenized limit analysis stochastic approach based on LatinHypercube sampling (LHS) with small population and response surface approximation (RSA) is used instead of expensive large scale Monte Carlo (MCS) simulations with the aim of reproducing buildings ultimate strength empirical cumulative distribution function (ECDF).

The method (-a) gives results in terms of collapse load in good agreement with large scale MCS results and (-b) allows a strong reduction of the total computational numerical effort, since only few FE limit analysis simulations are required.

When a fixed structural geometry and a given set of applied external loads is considered, the collapse load is only a function of the material mechanical properties at failure, mathematically formalized as:

$$
\lambda=g(\mathbf{x})
$$

where $\mathbf{x}=\left(x_{1}, x_{2}, \ldots, x_{m}\right)$ is the set of material input variables (e.g. cohesion, friction angle, masonry compressive strength, etc.).

Function $g(-)$ thus represents the mathematical model of the input-output relationship established by a limit state analysis of a given building. It should be emphasized that this relationship, even it might be non-linear, is strictly deterministic, since replicates the same output collapse loads when the same material input parameters are assumed. As the above equation show, the collapse load of a given structure strictly depends on the strength parameters chosen as input variables. As a consequence, when a homogenized limit analysis for a masonry building is performed, the material strength properties, as well as the resulting collapse load, should be treated as random quantities. This implies that it is necessary to account for all uncertainties, in material strength data, as well as in the calculated collapse load. In particular, we need appropriate probabilistic methodologies to estimate the probability distribution of the calculated random limit load of an existing building, to determine the resultant failure probability and the safety levels for each loading condition.

On the other hand, FE limit analyses of complex buildings are usually computationally very demanding even for a single run and may require many hours for a full sensitivity analysis. Therefore, it appears clear that large sample sizes 
required in the classical Monte Carlo with random sampling become computationally too expensive for practical applications.

Therefore, practitioners need strategies alternative to the pure Monte Carlo method, which would be capable both to reduce the overall computational time and to give the required statistical accuracy.

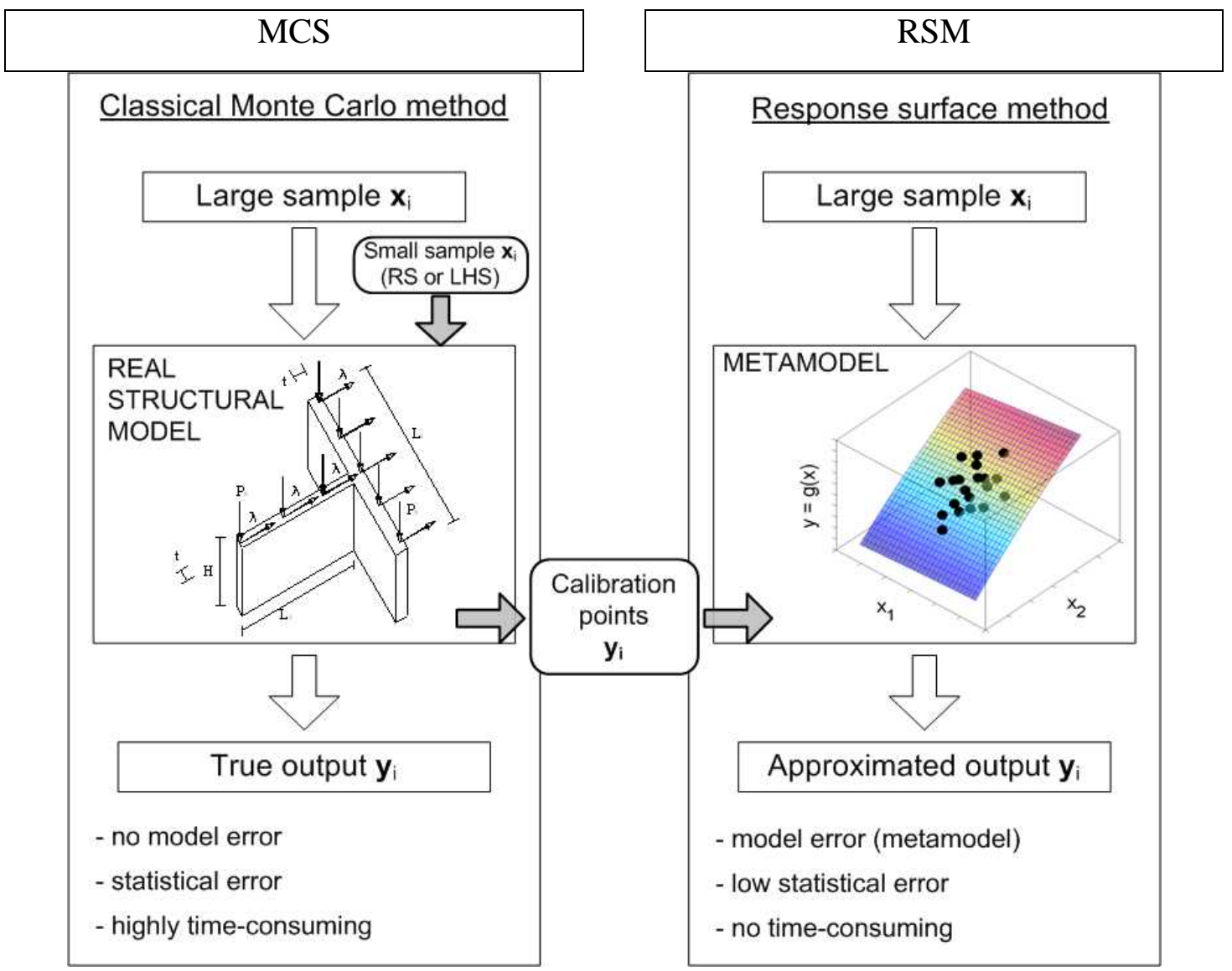

Figure 10: Schematic representation of direct Monte Carlo method and response surface method.

A possibility is to improve the sampling scheme by adopting other techniques, able to provide the same statistical convergence with smaller sample sizes. Among the existing techniques, the Latin Hypercube sampling has received in the last decades an increasing attention and application in many different research areas, when large samples are not computationally practicable.

In any case, even if improved sampling techniques, as the Latin Hypercube method (LH), can drastically reduce the total number of simulation runs required, in many cases of technical interest, the total time spent in computer simulations still remains very high. To further overcome this limitation, other methodologies have to be explored and applied in combination with LH sampling. In this framework, a promising approach is to use response surface methods (RSM) to approximate the 
input/output relationship in a time-consuming FEM analysis (formalized here by function $g(-)$ ), with a less-expensive suitable mathematical approximation.

In response surface methods (see Figure 10 and [26]-[28]) the true, but unknown, function $g(-)$ is replaced by an appropriate approximation $\hat{g}(-)$, which will be used as an inexpensive surrogate (or metamodel) of real FEM calculations in all input/output analysis computations. The surrogate model $\hat{g}(-)$ is calibrated on observed analysis outputs, resulting from calculations on a set of optimally selected input values.

Several studies, see for instance [26][27], have systematically investigated the capabilities of such methods and tried to establish what combination of sampling technique and response surface approximation would give the best level of accuracy.

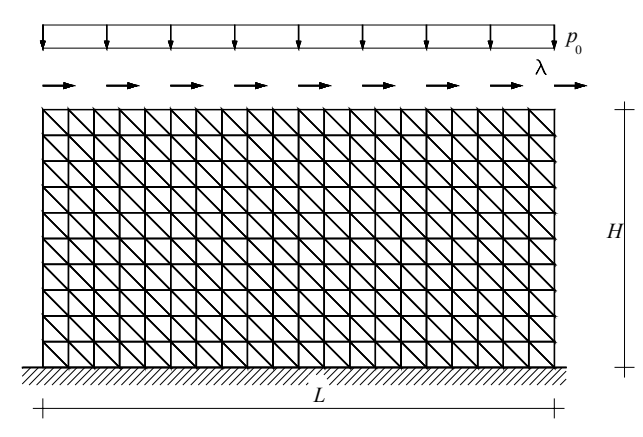

$-\mathrm{a}$

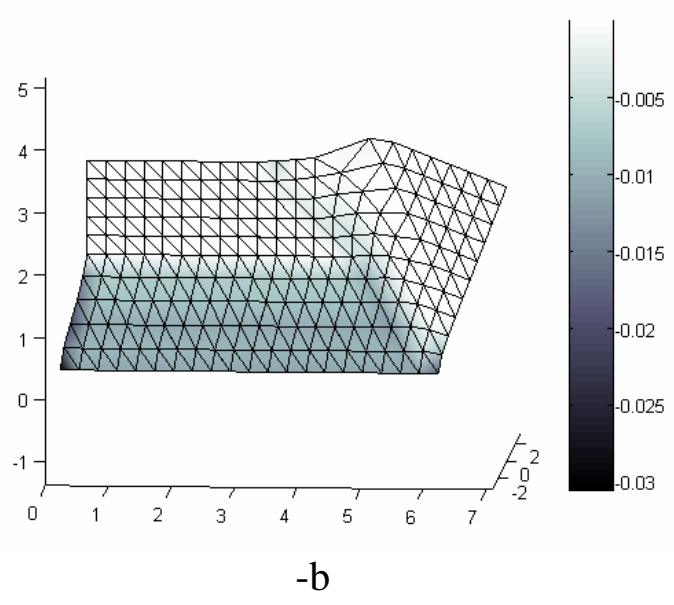

$-b$

Figure 11: -a: Shear wall with random input mechanical properties; mesh used for the limit analysis FE Monte Carlo simulations. -b: Typical deformed shape at collapse obtained with the limit analysis FE model.

Assuming that the approximation error is small and technically acceptable, the crucial advantage of this approach is that the minimum sample sizes required for a sufficiently accurate calibration can be very small, and drastically lower than those required by classical Monte Carlo analysis or alternative Latin Hypercube sampling.

As a structural example, let the shear wall of Figure 11 be considered, with dimensions $L x H x t=600 \times 300 \times 45 \mathrm{~cm}$ (length $\mathrm{x}$ height $\mathrm{x}$ thickness) and vertical load equal to $45 \mathrm{KN} / \mathrm{m}$, corresponding to a low average compressive stress equal to $0.1 \mathrm{~N} / \mathrm{mm}^{2}$. For the sake of simplicity, the wall is assumed build in stretcher bond with common Italian bricks of dimensions $250 \times 120 \times 55 \mathrm{~mm}$ infinitely resistant and joints reduced to interfaces with cohesive frictional behaviour and limited tensile strength $f_{t}$, see Figure 12. Mortar tensile strength $\left(f_{t}\right)$, cohesion $(c)$ and friction angle $(\tan (\Phi))$ are assumed as random variables. While for cohesion $c$ and $\tan (\Phi)$ normal distributions are adopted in agreement with experimental data reported in [29], for $f_{t}$ a typical limitation $f_{t} \leq c / \tan (\Phi)$, Figure 12, should be introduced, hence precluding the utilization of normal distributions. As a rule, a $i-t$ input 
point $\left(c_{i}, \tan \left(\Phi_{i}\right), f_{t}^{i}\right)$ randomly generated (supposing variables normally distributed), could result physically inadmissible, being the case $f_{t}^{i}>c_{i} / \tan \left(\Phi_{i}\right)$ possible.

In order to avoid such a possible inadmissibility of sampled values during large MC simulations, we assume that tensile strength depends both on $c$ and $\tan (\Phi)$ via an $a$ pivot random variable, according to the following relation:

$f_{t}=a \cdot c / \tan (\Phi)$

where $a$ is normally distributed with mean value equal to 0.5 .

It is worth noting that $a$ standard deviation is calibrated in order to obtain an output distribution for $f_{t}$ through equation (10) reproducing typical experimental data available in the literature.

Table III: Mean value and standard deviation for cohesion $c, \tan (\Phi)$ and $a$.

\begin{tabular}{|c|c|c|c|}
\hline & $\begin{array}{c}\text { Cohesion } \\
c\left[N / \mathrm{mm}^{2}\right]\end{array}$ & $\begin{array}{c}\text { Friction angle } \\
\tan (\Phi)\end{array}$ & $\begin{array}{c}\text { Variable } \\
a\end{array}$ \\
\hline Mean value $\mu$ & 0.1419 & 0.7515 & 0.5 \\
\hline $\begin{array}{c}\text { Standard } \\
\text { deviation } \sigma\end{array}$ & 0.0361 & 0.0469 & 0.036 \\
\hline $\operatorname{COV}[100 \sigma / \mu]$ & 25.44 & 6.24 & 7.21 \\
\hline
\end{tabular}

Following equation (10), mean values and standard deviations summarized in Table III for $a, c$ and $\tan (\Phi)$ stochastic variables are adopted. In Figure 13, the corresponding histograms for $f_{t}, c$ and $\Phi$ are reported. In particular, $f_{t}$ distribution (although obtained numerically) results in good agreement with consolidated experimental data available and with a typical Gaussian distribution.
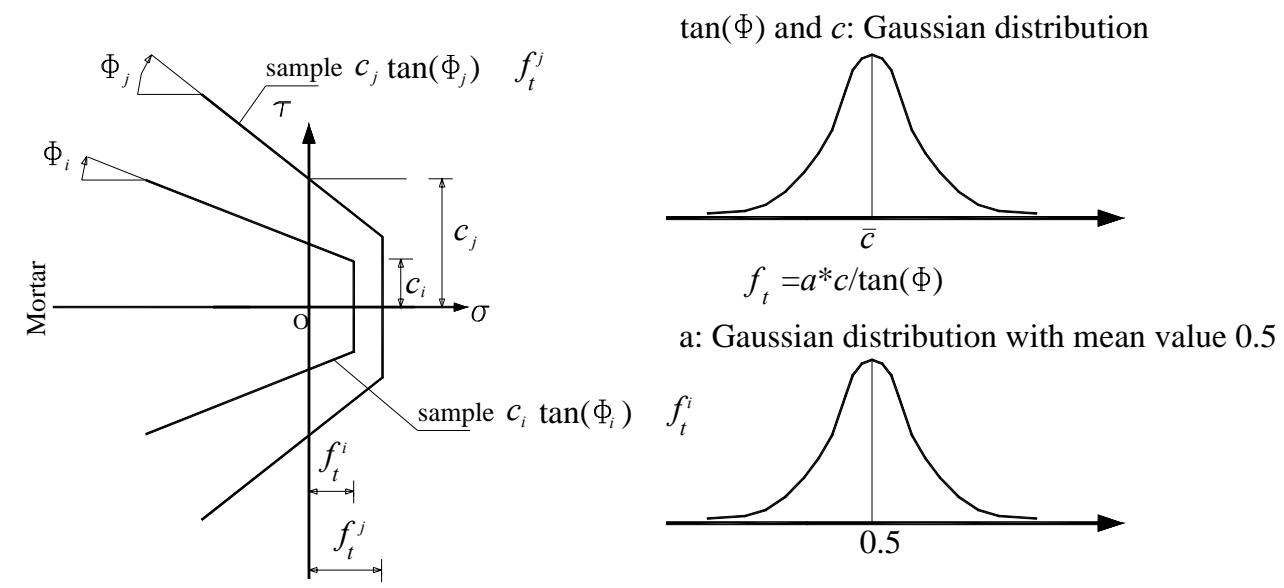

Figure 12: Probability distribution of stochastic variables $c, \tan (\Phi)$ and $a$. 
Four large scale Monte Carlo simulations with 1000 points are performed in order to reproduce the output distribution of collapse load obtained by means of limit analysis. A refined mesh with 400 triangular three-noded elements is used for the simulations, as shown in Figure 11.

Normal probability plots, useful for graphical normality testing, of the collapse load for each MC simulation are shown in

Figure 14. The Gaussian behaviour also for low values of the collapse load (corresponding to low values of $f_{t}$ and $c$ ) is worth noting. In particular, values of kurtosis for the collapse load distributions is around 3 for each MC simulation, demonstrating that the output stochastic variable is almost Gaussian.
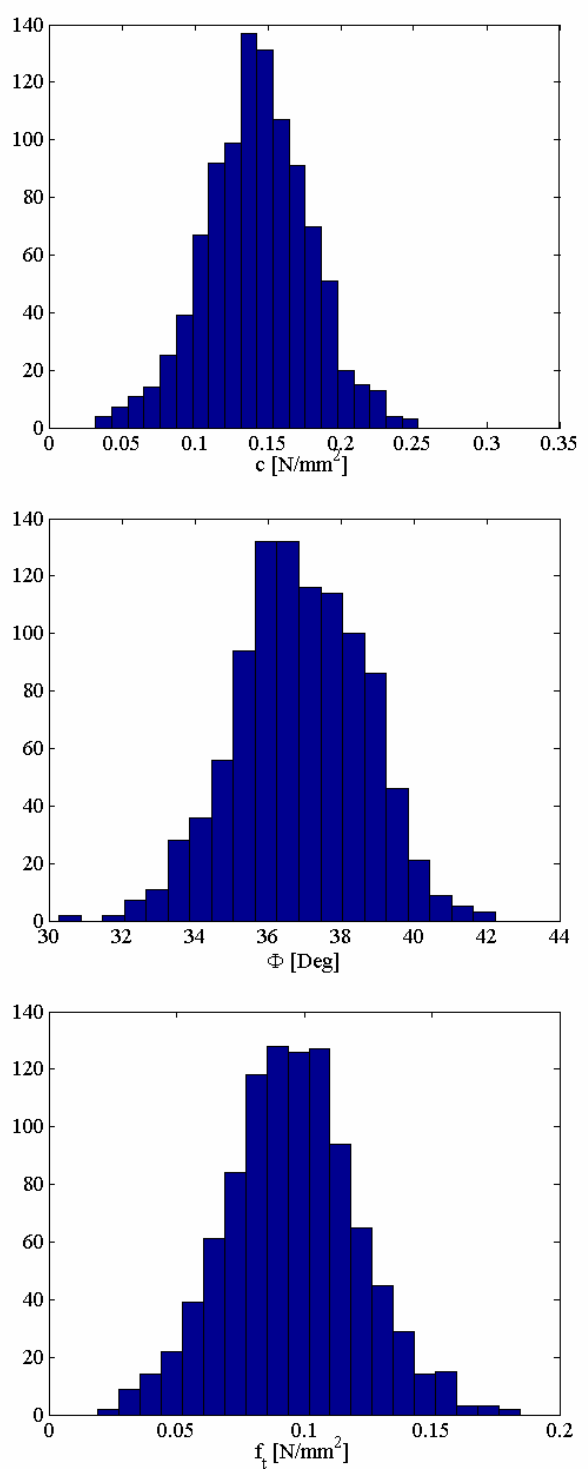

Figure 13: Histograms of $c(-\mathrm{a}), \Phi(-\mathrm{b})$ and $f_{t}$ variables (-c). 
It is worth noting that each of the 1000 points MC simulations required approximately 12 hours to be performed on a Intel Pentium $3 \mathrm{GHz}$ PC equipped with $1 \mathrm{~Gb}$ RAM.

In order to reduce drastically computational costs, a number of different response surface methods (RSM) available in the literature for complex deterministic analysis systems can be used to design surrogate models. The difference among all the existing techniques (e.g. polynomial regression, kriging, multivariate adaptive regression splines, etc.) relies in their relative accuracy and complexity. Among all, the most popular surrogate model is probably the polynomial regression method, being calculations trivial and the resulting approximation expressed in closed-form.

Let $y=g(\mathbf{x})$ the true response corresponding to a given sample $\mathbf{x}=\left(x_{1}, x_{2}, \ldots, x_{m}\right)$ of input random variables. A second order polynomial approximation is written as:

$$
\hat{y}(\mathbf{x})=c_{0}+\sum_{i=1}^{m} c_{i} x_{i}+\sum_{i=1}^{m} \sum_{j=i+1}^{m} c_{i j} x_{i} x_{j}+\sum_{i=1}^{m} c_{i i} x_{i}^{2}
$$

where $m$ is the total number of design variables (i.e. number of random material parameters), $x_{i}$ is the $i$-th design variable (random material property) and $c_{i}$ is the set of unknown coefficients.

Let $\mathbf{y}=\left[y^{(1)}, y^{(2)}, \ldots, y^{(n)}\right]$ the vector of $n$ observed values, corresponding to the $n$ samples of the input random variables $\mathbf{x}$. The unique lest-squares solution is given as:

$$
\hat{\mathbf{c}}=\left(\mathbf{X}^{T} \mathbf{X}\right)^{-1} \mathbf{X}^{T} \mathbf{y}
$$

where:

$$
\mathbf{X}=\left[\begin{array}{ccccc}
1 & x_{1}^{(1)} & x_{2}^{(1)} & \ldots & \left(x_{n v}^{(1)}\right)^{2} \\
\vdots & \vdots & \vdots & \ddots & \vdots \\
1 & x_{1}^{(n S)} & x_{2}^{(n S)} & \ldots & \left(x_{n v}^{(n S)}\right)^{2}
\end{array}\right]
$$

is the matrix constructed from the sample of design variables.

Even if the use of polynomial approximation is quite simple, it is worth noting that in the fitting procedure of the model in (12), all observed values are equally weighted, hence the prediction at an unsampled location depends "globally" on all observed values.

For the problem at hand, a quadratic polynomial approximation $\hat{g}(-)$ of the true complex system response $y=g(\mathbf{x})$ is constructed based on calibration points generated by either random or Latin Hypercube sampling. Variability in metamodel generation is evaluated by constructing 8 independent replicates (that is, 8 independent surrogate functions $\hat{g}(-))$.

For each MC or LH input points $\left(c_{i}, \tan \left(\Phi_{i}\right), f_{t}^{i}\right)$, a 3D homogenized limit analysis is performed, obtaining each time an output value of the collapse load $\lambda$. 


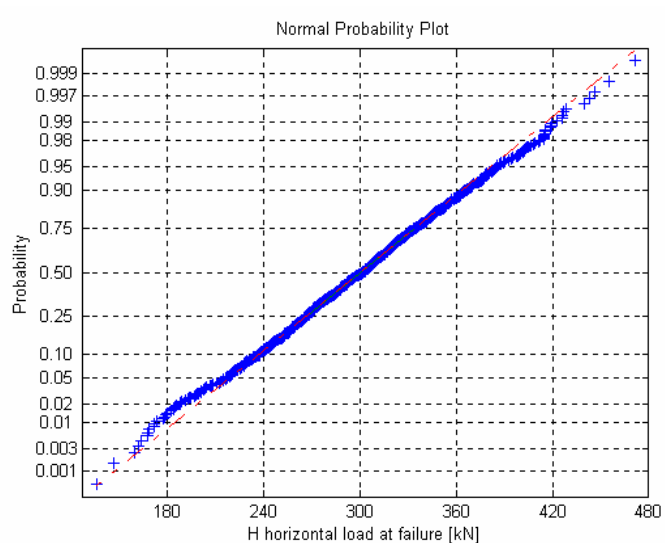

Monte Carlo simulation 1 (kurtosis collapse load $\lambda=3.14$; kurtosis $c=3.10$; kurtosis $\tan (\Phi)=3.01$; kurtosis $f_{t}=2.77$, time required for the 1000 points simulations 12 hours 15 minutes)

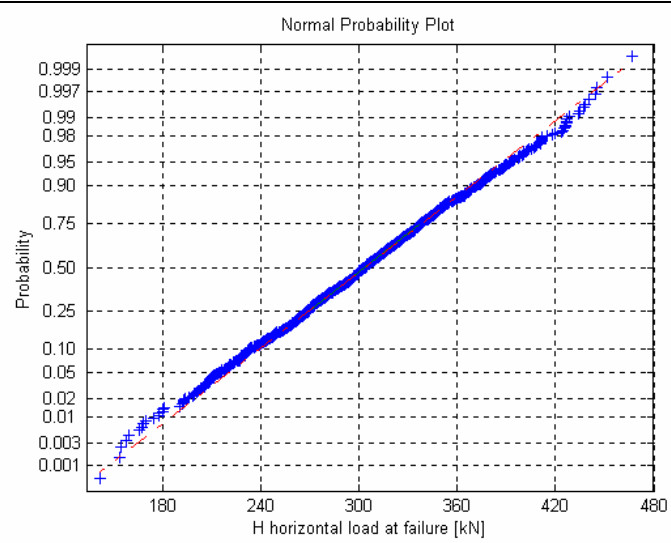

Monte Carlo simulation 3 (kurtosis collapse load $\lambda=3.06$; kurtosis $c=2.80$; kurtosis $\tan (\Phi)=3.14$; kurtosis $f_{t}=2.86$, time required for the 1000 points simulations 12 hours 00 minutes)

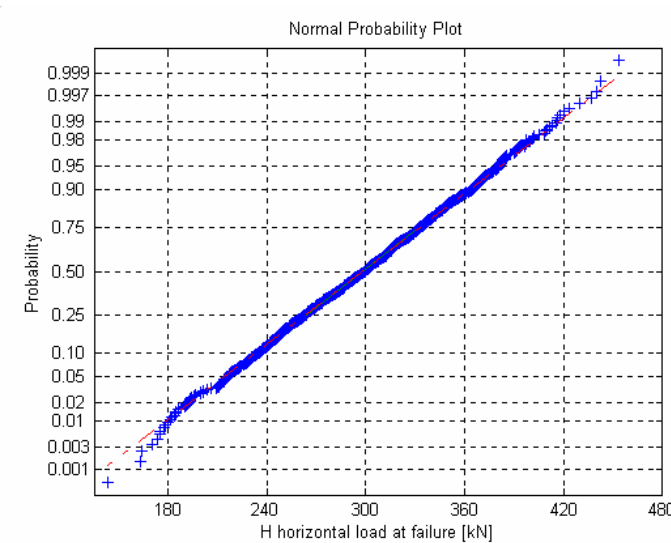

Monte Carlo simulation 2 (kurtosis collapse load $\lambda=2.85$; kurtosis $c=2.88$; kurtosis $\tan (\Phi)=3.07$; kurtosis $f_{t}=2.95$, time required for the 1000 points simulations 13 hours 1 minutes)

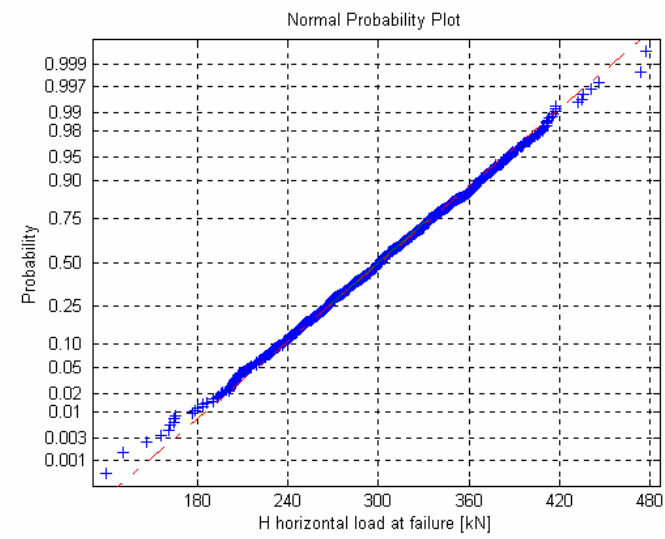

Monte Carlo simulation 4 (kurtosis collapse load $\lambda=3.12$; kurtosis $c=2.98$; kurtosis $\tan (\Phi)=3.25$; kurtosis $f_{t}=3.01$, time required for the 1000 points simulations 13 hours 43 minutes)

Figure 14: Results from four Monte Carlo simulations with 1000 elements.

In particular, with the data collected from the simulations, 16 different surrogate surfaces for the collapse load are obtained, respectively 8 surfaces from direct MC simulations with 30 replicates and 8 surfaces from LH simulations with 30 replicates.

As already mentioned, no information is available on the actual $\lambda\left(c, \tan (\Phi), f_{t}\right)$ relationship, meaning that the true cumulative distribution is approximated by pooling the observed values corresponding to the four sets of large scale Monte Carlo simulations. 
In Figure 15-a, a comparison between the mean absolute relative error (called here "MARE indicator" and defined as MARE $=\frac{1}{n} \sum_{i=1}^{n}\left|\frac{y_{i}-\hat{y}_{i}}{y_{i}}\right|$, where $n=8$ is the number of metamodels replicates and $\hat{y}_{i}$ is the collapse load evaluated on the surrogate function), obtained from $8 \mathrm{LH}$ and $8 \mathrm{MC}$ metamodels is shown. As it is possible to note, metamodels constructed from LH samples are more accurate with respect to MC ones, especially for low probability values. Furthermore, it is clearly evident the increasing trend of the error for both models reducing collapse load from 300 to $120 \mathrm{kN}$.
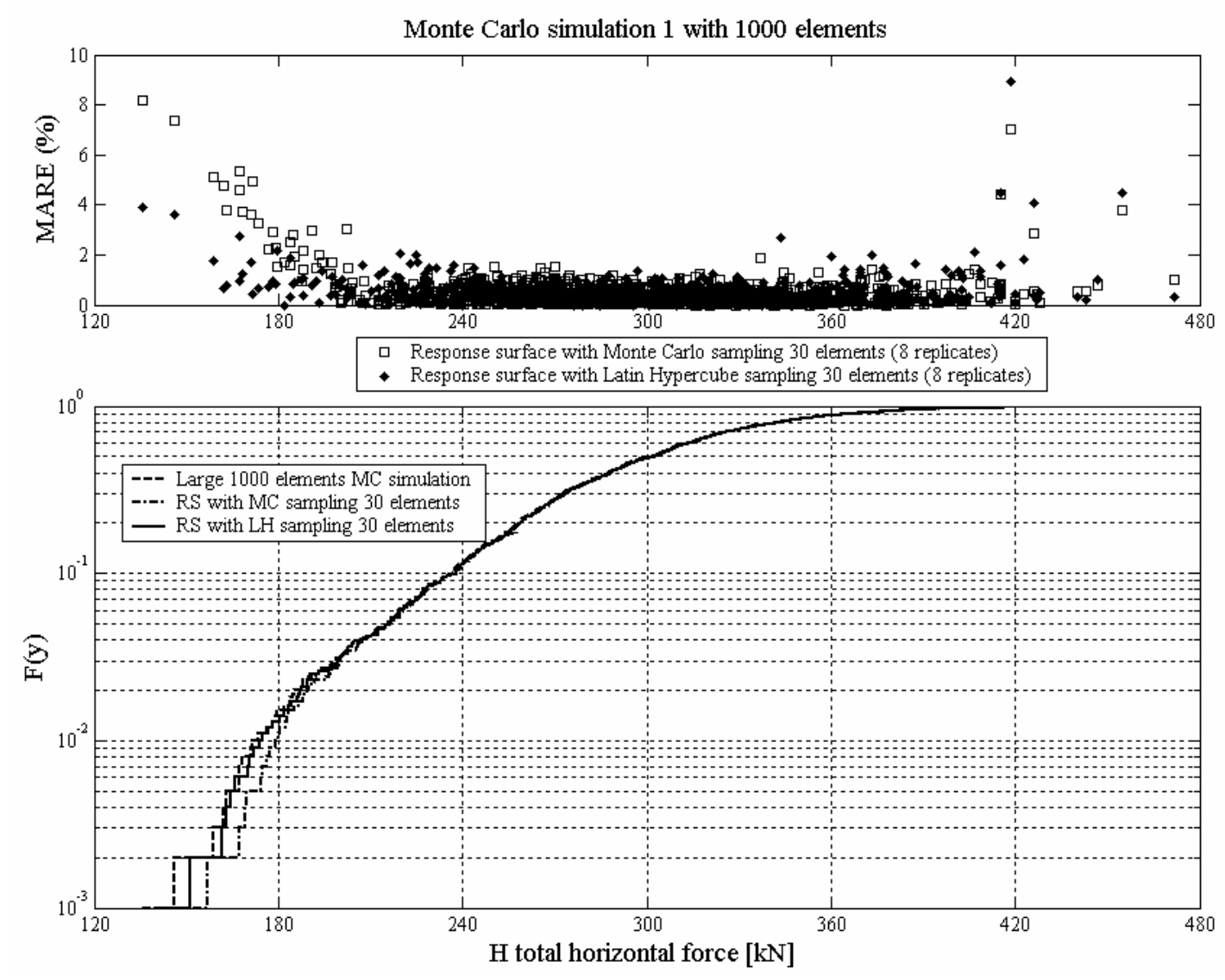

Figure 15: Comparison between metamodels from LH sampling and MC sampling with 30 points on large MC simulation 1 (1000 points). LH sampling gives a CDF more accurate with respect to MC for low probability values.

In Figure 15-b, a comparison among CDF obtained with LH metamodels, MC metamodels and empirical CDF from a large MC simulation with 1000 points is reported. Cumulative distributions confirm MARE indicator predictions of Figure 15-a, i.e. that LH metamodels fit quite accurately large MC simulation results for low values of probability. On the other hand, Figure 15 results underline that the quality of the surrogate functions near the boundary of the domain sensibly deteriorates. Such a behaviour is due to the fact that least square polynomial 
approach fits data well in the range where input variables are randomly generated. As a rule, random small populations do not cover sufficiently regions corresponding to small values of the output variable. Consequently, LH sampling generally is more accurate than $\mathrm{MC}$, being input variables suitably selected on sub-regions with equal probabilities.

Finally, it is worth noting that the approach based on surrogate functions could be attractive from an engineering point of view when reliability analyses on complex structures are required. The use of surrogate functions, in fact, allows to have at disposal output quantities quickly and with a very limited computational effort if compared with direct large MC simulations. In combination with LH sampling on input variables, the surrogate surfaces method is also able to reduce inaccuracies of the output response at low probability values. On the other hand, the quality of the results obtained is in any case affected by increasing errors at low probabilities. Furthermore, it is worth mentioning that the actual distribution of the collapse load for real structures is usually unknown. Therefore, no information on the error committed using metamodels is at disposal.

\section{Conclusions}

In the present paper, a homogenization limit analysis model based on a plate and shell upper bound FE formulation has been presented. In the model, the elementary cell is subdivided along its thickness into several layers. For each layer, fully equilibrated stress fields are assumed, adopting polynomial expressions for the stress tensor components in a finite number of sub-domains. The continuity of the stress vector on the interfaces between adjacent sub-domains and suitable anti-periodicity conditions on the boundary surface are further imposed. In this way, linearized homogenized surfaces in six dimensions (polytopes) for masonry in- and out-ofplane loaded are obtained. Such surfaces are then implemented in a FE limit analysis code for the analysis at collapse of entire 3D structures and meaningful examples of technical relevance are discussed in detail.

The micro-mechanical model presented competes favorably with more traditional approaches, such as for instance full 3D heterogeneous techniques and "at hand" calculations based on the assumption of zero resistance of masonry in tension. In fact, full 3D analyses performed on entire buildings by means of the homogenization model presented, require a reduced computational cost (less than 150 seconds for a single optimization reported in Section 4), do not require an a-priori evaluation of the collapse mechanisms and can take into account important features of masonry at failure. Furthermore, limit analysis is able to give important information at failure, such as failure mechanisms, collapse loads, stress distribution, plastic dissipation zones, etc.. Therefore, the model presented results a valuable tool for practitioners involved in advanced analyses of full 3D masonry structures subjected to seismic actions.

The limited computational effort of the optimization problems obtained in this framework allows to tackle interesting engineering problems, such as for instance the evaluation of collapse loads stochastic distribution of masonry structures when 
mortar mechanical properties (i.e. input parameters) are assumed as random variables.

The combination of homogenization, limit analysis and response surface approximation allows to obtain reliable predictions of failure loads distribution when metamodels are built starting from few points sampled randomly and making use of both traditional Monte Carlo approaches and Latin Hypercube sampling. In this way, a reliable estimation of output collapse loads distribution can be obtained avoiding to perform expensive Monte Carlo simulations with several points.

\section{Acknowledgements}

Paulo B. Lourenço acknowledges partial funding from project POCI-ECM-616712004, "Seismic vulnerability reduction of old masonry buildings", from Portuguese Science and Technology Foundation (FCT).

\section{References}

[1] P.B. Lourenço, "Experimental and numerical issues in the modeling of the mechanical behavior of masonry”, In Roca P. et al. (ed.), Structural analysis of historical constructions II, p. 57-91. CIMNE, Barcelona, 1998.

[2] P.B. Lourenço, “Computations of historical masonry constructions”, Progress in Structural Engineering and Materials, 4(3), 301-319, 2002.

[3] L. Ramos, P.B. Lourenço, "Modeling and vulnerability of historical city centers in seismic areas: a case study in Lisbon”, Engineering Structures, 26, 1295-1310, 2004.

[4] A. Giuffrè (editor), "Safety and conservation of historical centers: the Ortigia case”, Laterza Press, Roma - Bari, 1993 [in Italian].

[5] O.P.C.M. 3274, 20/03/2003, "First elements concerning general criteria for the seismic classification of the national territory and technical norms for structures in seismic zone" [in Italian], Primi elementi in materia di criteri generali per la classificazione sismica del territorio nazionale e di normative tecniche per le costruzioni in zona sismica.

[6] O.P.C.M. 3431/05 09/05/2005, "Further modifications and integrations on OPCM 3274/03” [in Italian], Ulteriori modifiche ed integrazioni all'OPCM 3274/03.

[7] P.B. Lourenço R. de Borst , J. Rots, "A plane stress softening plasticity model for orthotropic materials”, International Journal for Numerical Methods in Engineering, 40, 4033-4057, 1997.

[8] P.B. Lourenço, J. Rots, J. Blaauwendraad, "Continuum model for masonry: Parameter estimation and validation”, J. Struct. Engrg., ASCE, 124(6), 642652, 1998.

[9] A. Zucchini, P.B. Lourenço, "A micro-mechanical model for the homogenisation of masonry”, International Journal of Solids and Structures, 39, 3233-3255, 2002. 
[10] P. Suquet, “Analyse limite et homogeneisation”, Comptes Rendus de l'Academie des Sciences - Series IIB - Mechanics, 296, 1355-1358, 1983 [in French].

[11] G. Milani, P.B. Lourenço, A. Tralli, "Homogenised limit analysis of masonry walls. Part II: structural examples”, Comp. Struct., 84, 181-195, 2006.

[12] G. Milani, P.B. Lourenço, A. Tralli, "Homogenised limit analysis of masonry walls. Part I: failure surfaces”, Comp. Struct., 84, 166-180, 2006.

[13] G. Milani, P.B. Lourenço, A. Tralli, "Homogenization approach for the limit analysis of out-of-plane loaded masonry walls”, ASCE Journal of Structural Engineering, 132 (10), 1650-1663, 2006.

[14] G. Milani, P.B. Lourenço, A. Tralli, "Homogenized limit analysis of masonry buildings under horizontal loads", in press in Engineering Structures.

[15] S.W. Sloan, P.W. Kleeman, "Upper bound limit analysis using discontinuous velocity fields”, Computer Methods in Applied Mechanics and Engineering, 127(1-4), 293-314, 1995.

[16] J. Munro, A.M.A. Da Fonseca, "Yield-line method by finite elements and linear programming”, ASCE Journal of Structural Engineering, 56B, 37-44, 1978.

[17] A.A. Cannarozzi, M. Capurso, F. Laudiero, "An iterative procedure for collapse analysis of reinforced concrete plates”, Computer Methods in Applied Mechanics and Engineering, 16, 47-68, 1978.

[18] K. Krabbenhoft, A.V. Lyamin, M. Hjiaj, S.W. Sloan, “A new discontinuous upper bound limit analysis formulation”, International Journal for Numerical Methods in Engineering, 63, 1069-1088, 2005.

[19] S.W. Sloan, "A steepest edge active set algorithm for solving sparse linear programming problems", International Journal Numerical Methods Engineering, 12, 61-67, 1988.

[20] P.C. Olsen, "Rigid-plastic finite element analysis of steel plates, structural girders and connections”, Computer Methods in Applied Mechanics and Engineering, 191, 761-781, 2001.

[21] P.B. Lourenço, “Computational strategies for masonry structures”, Ph.D. Thesis Delft University of Technology, the Netherlands 1996. Available at www.civil.uminho.pt/masonry.

[22] P.B. Lourenço, J. Rots, "A multi-surface interface model for the analysis of masonry structures” ASCE Jour. Eng. Mech., 123(7), 660-668, 1997.

[23] D.M. 16/01/1996 (G.U. 5-2-1996, N. 29), "Technical norms relative to general criteria for the safety assessment of structures and loads" [in Italian], Norme tecniche relative ai "Criteri generali per la verifica di sicurezza delle costruzioni, e dei carichi e sovraccarichi”.

[24] A. Brencich, L. Gambarotta, S. Lagomarsino, "Catania project: research on the seismic response of two masonry buildings. Chapter 6: Analysis of a masonry building in via Martoglio”, In: CNR Gruppo Nazionale per la Difesa dei Terremoti (editor), 107-151, 2000 [in Italian].

[25] G. Magenes, C. Braggio. "Catania project: research on the seismic response of two masonry buildings. Chapter 7: analysis of a masonry building in via 
Martoglio. In: CNR Gruppo Nazionale per la Difesa dei Terremoti (editor), 153-190, 2000 [in Italian].

[26] R. Jin, W. Chen, T.W. Simpson, "Comparative studies of metamodeling techniques under multiple modeling criteria”, 8th AIAA/NASA/USAF/ISSMO Symposium on Multidisciplinary Analysis and Optimization, Long Beach, CA, paper AIAA-2000-4801, 2000.

[27] T.W. Simpson, J.D. Peplinski, P.N. Koch, J.K. Allen, "Metamodels for Computer-based Engineering Design: Survey and recommendations", Engineering with Computers, 17, 129-150, 2001.

[28] P. Towashiraporn, "Building seismic fragilities using response surface metamodels”, Ph.D. Thesis, Georgia Institute of Technology, U.S.A. 2004.

[29] R. van der Pluijm R, "Out-of-plane bending of masonry. Behaviour and strength", Ph.D. Thesis, Eindhoven University of Technology, the Netherlands 1999. 